\title{
The Prevalence of Neoplasm Diseases and Investigation of Some Biochemical Serum Parameters
}

\author{
Hasan Karagecili' ${ }^{(D m}$ Emah Yerlikaya ${ }^{\text {(D) }}$ Mustafa Oguzhan Kaya ${ }^{3}$ (D) \\ ${ }^{1}$ Siirt University, Department of Nursing, Siirt, Turkey \\ ${ }^{2}$ Siirt University, Department of Nutrition and Dietetics, Siirt, Turkey \\ ${ }^{3}$ Kocaeli University, Department of Chemistry, Kocaeli, Turkey
}

\section{A B STR ACT}

A im: We aimed in this work to investigate the skin, lung, and bone marrow-related cancer prevalence and biochemical serum parameters of all these cancer patients.

Materials and Methods: The skin, lung, and bone marrow cancer patient's biochemical serum data examined retrospectively using a hospital information system.

Results: Basal cell carcinoma patients number recorded as 155 with $63 \%$, squamous cell carcinoma patients number were enrolled in 73 with $30 \%$ and, malign melanoma patients number noted as 10 with $4 \%$. Skin cancers were the most seen cancer type in 246 patients with 22\% between 2013-2017 years. All cancer patient numbers were 1134 between these years. The common incidences of basal cell carcinoma and squamous cell carcinoma cancers observed on the upper part of the body, respectively on the face-cheek, nose, ear, eyelid, and lip. In this study, there was a significant difference among skin cancer patient's serum glucose, aspartate aminotransferase, alanine aminotransferase, sodium, bilirubin direct, bilirubin total, creatinine, urea level, and control group serum parameters level, $\mathrm{p}<0.05$. The lung cancer patient numbers were 119 with $10.4 \%$, bone marrow patients numbers were 113 with $10 \%$. Two cancer groups were statistically different in terms of 5 -years survival. $\log \operatorname{Rank} \mathrm{X}^{2}=8.68, \mathrm{p}<0.05$.

Conclusions: We recorded that skin cancer types and regions on the upper parts of the body were more because of exposure to the sun. The lung cancer survival rate was lower than bone marrow cancer. Moreover, we strongly emphasized that measuring the biochemical serum parameters was statistically significant with the diagnosis of cancer patients.

\author{
Article History: \\ Received: 2021/04/20 \\ Accepted: 2021/06/02 \\ Online: $2021 / 06 / 30$
}

Correspondence to: Hasan Karagecili, Siirt University, Faculty of Health, Department of Nursing, 56100 Siirt, Turkey E-Mail: hasankaragecili@siirt.edu.tr Phone: $+90(484) 2231224$

Fax:+9o (484) 2235156

\section{Keywords:}

Carcinoma; Malignant; Skin cancer; Lung; Incidence; Bone marrow.

\section{INTRODUCTION}

ancer is a very complex illness happening via ge$\checkmark$ netic variation or anomaly that allows the conversion of healthy cells into disordered cells, which results in interaction among the genetic factors and environmental factors [1]. Skin is the prevalent malignant neoplasms site. Skin cancers have a higher incidence than others in summation [2]. Skin cancer constitutes a prevalent group of malignant neoplasms among white people. The frequency of melanoma and non-melanoma skin cancer (NMSC) increased throughout the world [3]. Skin cancers include basal cell carcinoma (BCC) and squamous cell carcinoma (SCC), generally called NMSC, and melanoma skin cancers are the most recurrent cancer both in America and in other developed countries with mostly fairskinned people [4]. Melanoma is the utmost mortal type of skin cancer and has turned into one of the widespread cancers among people in their teens in
Canada, especially for women. Among all NMSC examples, almost $77 \%$ are BCC, and $23 \%$ are SCC. Even though seldom mortal, the high ratio of NMSC indicates a notable load of illness in the way of morbidity, the standard of living, social effect, and healthcare expenditures [5]. The incidences of skin cancers were found high in Australia. It has four times more prevalence than other whole cancers [6]. Melanoma and NMSC's risk factors include exposure to ultraviolet (UV) radiation and the susceptibility of a person's skin to UV radiation [7]. Also to X-rays, Human papilloma virüs, arsenic compounds, and other chemical products [8]. The commonly seen region of NMSC is on the head and neck area, and surgery remains the mainstay of treatment [9]. Lung cancer is the most common cancer in the world. It is one of the deadliest and most aggressive types of cancer, with a 5 -years survival rate of barely $15 \%$. Every year 1.7 
million people die of lung cancer in the world. 80-90\% of lung cancer cases are due to smoking, making lung cancer one of the most preventable cancer types [10]. The two main types of lung cancer are non-small cell lung cancer (NSCLC) and small cell lung cancer (SCLC). There are three types of NSCLC; $40 \%$ of the cases are adenocarcinoma, 30\% of squamous cell carcinoma (SCC), and $15 \%$ of large cell carcinomas. The Small cell lung cancer rate is $15 \%$. These tumors are classified by histology, and as the size of biopsy specimens becomes smaller, the assessment of immunohistochemistry plays an important role [11]. Bone marrow is the primary hematopoietic organ involved in many malignant diseases, include acute and chronic leukemia, multiple myeloma, myelodysplastic syndrome, and bone metastasis from solid tumors [12] We aimed to identify the incidence of skin cancers, lung cancers, and bone marrow cancers, according to type in the Siirt province. And to compare the incidence of them with other regions, with this analysis at first, to provide up-to-date prevalence approximate by sex, age, body site, survival; and second, to research the levels of some serum parameters in cancers patients and healthy person comparatively.

\section{MATERIALS AND METHODS}

Before starting to work, both the Public Health Directorate and the Siirt State hospital preauthorization documents were about the feasibility of the work. In this study, the files of patients who applied to Siirt State Hospital and Siirt Public Health units and were diagnosed with a neoplasm or malign were between 2013-2017. Patients were diagnosed with neoplasm constituted the experimental group, and healthy subjects were the control group. The study is a retrospective study. The files of the patients were diagnosed with neoplasm were applied to the polyclinics evaluated. Our work was on women and men. The study was composed of experimental and control groups. The experimental group consisted of patients with various neoplasms (skin cancers, lung cancer, bone marrow cancer), and the control group consisted of healthy subjects who received routine control. Fasting blood glucose, amylase, alanine aminotransferase (ALT), aspartate aminotransferase (AST), lactate dehydrogenase (LDH), electrolytes values, and biochemical findings retrospectively took from patient files. Biochemical parameters studied in the biochemistry laboratory of Siirt State Hospital using Advia autoanalyzer 1800. Cancer statistics collected with an active cancer registry system in many countries of the world. While the active cancer registry was in only two provinces of our country in 2002, it was put into practice in 81 Provinces as of 2013. Statistical analyzes performed using the SPSS 21. 0 statistical program for Windows to evaluate the data obtained from patient files and the hospital information system. Independent student $t$-test was used for parametric tests when data distributed normally. P values were less than 0.05 were assigned statistically.

\section{RESULTS}

Skin cancers: In 2013, the number of skin cancer patients was 66 in 235 cancer cases, and the skin cancer rate was $28 \%$. In 2014, the number of cancer patients was 321 , and the number of skin cancer patients number was 57 , and the rate was $17.7 \%$. In 2015, the number of cancer patients was 314 , and the number of skin cancer cases was 52 and 16.5\%. In 2016, the number of cancer patients was 124, and in 36 skin cancer cases detected, and the rate was $29 \%$. In 2017, the number of cancer patients recorded 140, and the ratio of skin cancer's in all cancers was 25\%. In 2013, the number of patients with BCC 37; 21 males and 16 females, 23 patients with SCC; 14 males and nine females. Three male malignant melanoma patients. The number of BCC patients in 2014 was 31; 17 males and 14 females, Number of SCC patients 20; 14 males and six females. It was two males and one female malignant melanoma patient. The number of BCC patients in 2015 was 34; 19 males and 15 females, the number of SCC patients was 19; 14 males and five females. one male and one female malignant melanoma patient recorded. The number of patients with BCC in 2016 was 25; 12 male and 13 female, the number of SCC patients were six; four males and two females. One male malignant melanoma, one male Kaposi's sarcoma, and one female Kaposi's sarcoma were. In 2017, the number of patients with basal cell cancer was 28; 13 males and 15 females, number of SCC patients were five; three males and two females. One female malignant melanoma was. Among the related years, 143 male skin cancer patients and 103 female skin cancer patients were. The overall skin cancer rate in men was $58 \%$, while the skin cancer rate in women was $42 \%$. Blood serum data of patients with malignant melanoma, $\mathrm{BCC}$, and SCC cancer evaluated retrospectively using a hospital information system. The incidence of patients determined. The number of BCC patients was 155 with $63 \%$, and the number of SCC patients was 73 with $30 \%$, and the number of patients with malignant melanoma was 10 with $4 \%$. The most common type of cancers are skin cancers, and the rate of it among five years in all cancers types recorded as $22 \%$ with a total of 246 patients. Non-melanoma, the most common skin cancers; the most common incidences of BCC and SCC cancers was observed on the upper part of the body, respectively on the face-cheek, nose, ear, eyelid, and lip at table 1 .

The number of skin cancers was observed in approximately 49 patients each year. The incidence of skin cancers 
Table 1. The incidence of skin cancer in the body region of patients.

\begin{tabular}{lcccccc}
\hline Location & $\begin{array}{c}\text { BCC } \\
n\end{array}$ & $\%$ & $\begin{array}{c}\text { SCC } \\
n\end{array}$ & $\begin{array}{c}\text { Total } \\
n\end{array}$ & \% Total \\
\hline Nose & 23 & 17.5 & 5 & 3.8 & 28 & 21.3 \\
Face-Cheek & 29 & 22.1 & 6 & 4.5 & 35 & 26.7 \\
Ear & 13 & 9.9 & 10 & 7.6 & 23 & 17.5 \\
Lip & 6 & 4.5 & 9 & 6.8 & 15 & 11.4 \\
Neck & 6 & 4.5 & 2 & 1.5 & 8 & 6.1 \\
Eye Lid & 19 & 14.5 & 3 & 2.2 & 22 & 16.7 \\
Total & 96 & 73 & 35 & 27 & 131 & 100 \\
\hline
\end{tabular}

was detected in almost 16 patients in the 100000 population in Siirt. All cancer incidence found as 76 patients in 100000 population. Between the given years the number of all cancer patients was calculated as 1134 years. Serum parameters levels of skin cancer patients compared with the control group serum parameters. In this study, skin cancer patient's serum glucose, AST, ALT, sodium, bilirubin direct, bilirubin total, creatinine, urea level were statistically significantly different compared with the control group's serum parameters levels, $\mathrm{p}<0.05$. It can be seen below from the group's statistics, table 2 .

Lung and bone marrow cancers, In 2013, 23 out of 235 cancer cases, 9.8\% were Lung cancer. The number of bone marrow cancer patients was 19 and 8\%. In 2014, the number of cancer patients was 321, and the number of lung cancer cases was 38 , with a rate of $11.8 \%$. Bone marrow cancer cases were 35 and 11\%. In 2015, the number of cancer patients was 312 , and the number of lung cancer cases was 37 and $16.5 \%$. Bone marrow cancer cases were 31 and 9.6\%. In 2016, the number of cancer patients was 124, and Lung cancer detected in 12 cases, the rate was $9.7 \%$. Bone marrow cancer cases are 12 and 9.7\%. In 2017, the number of cancer patients recorded 140, the number of lung cancers among all cancers was nine, and their rate was $6.4 \%$. Bone marrow cancer cases were 16 and 11.4\%. 2013 Number of lung cancer patients was 23; 17 Male and 6 Female, Number of bone marrow cancer patients were 19; 14 Men and 5 Women. 2014 Number of lung cancer patients was 38; 30 Male and 8 Female, Number of bone marrow cancer patients were 35; 22 males and 13 females. 2015 Number of lung cancer patients was 37; 31 Male and six females, Number of bone marrow cancer patients were 31; 23 Men and 8 Women. 2016 Number of lung cancer patients was 12; six males and six females, number of bone marrow cancer patients were 12; eight males and four females. 2017 Number of lung cancer patients was 9; nine Mae. The number of bone marrow cancer patients was 16; eight male and eight females. Between 2013 and 2017, 93 male lung cancer patients and 26 female lung cancer patients registered. The proportion of the total number of lung cancer patients seen in men was $78 \%$, and the proportion of

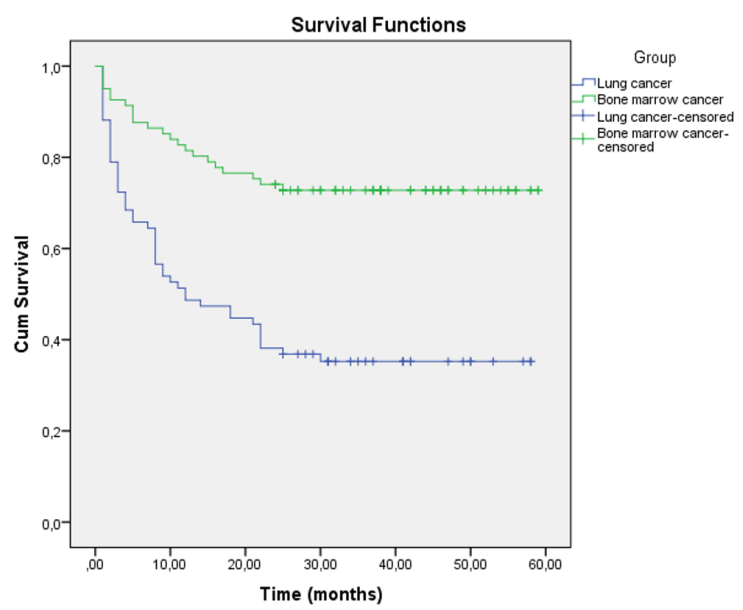

Figure 1. Kaplan-Meier, survival of lung cancer and bone marrow cancer patiens between 2013-2017 years.

women was 22\%. Between 2013-2017, 75 male bone marrow cancer patients and 38 female bone cancer patients registered. The proportion of bone marrow cancer patients in the total number of patients with bone marrow cancer was $66.4 \%$, and the rate of women was $33.6 \%$. The number of lung cancer patients was recorded as 119 to $10.4 \%$, while the number of bone marrow patients was 113 to $10 \%$. The incidence of lung cancer was approximately 24 each year, and the number of bone marrow cancer patients was 23 each year. The number of all cancer patients calculated as 1134 between these years. The number of patients with lung cancer was 76, and the number of patients with bone marrow cancer was 81.49 (64.5\%) of lung cancer patients, and 22 (27.2\%) of bone marrow cancer patients died between these years. Kaplan-Mier test shows the comparison of two disease group's statistical differences within using long rank, Breslow, and Tarone-Ware tests. p<0,05. Fig. 1.

In 2013, for 5-year survival analysis, the number of patients with lung cancer was 23 , and the number of patients with bone marrow cancer was 19. 16 (69.6\%) of the lung cancer patients and 4 (21.1\%) of the bone marrow cancer patients died. Two cancer groups were statistically different in terms of 5-years survival according to three tests. Graph 1. Kaplan-Miear survival analysis. Log Rank $\mathrm{X}^{2}=8.68, \mathrm{p}=$ $0.001, \mathrm{p}<0.05$. Breslow $\mathrm{X}^{2}=7,249, \mathrm{p}=0.007, \mathrm{p}<0.05$. TaroneWare $\mathrm{X}^{2}=8.009, \mathrm{p}=0.005, \mathrm{p}<0.05$. Fig. 2 .

In this study, there was a statistically significant difference between lung cancer and bone marrow cancer patient's serum glucose, AST, GGT, LDH, urea level compared with the control group's serum parameters levels, $\mathrm{p}<0.05$. Cancer patient's biochemical serum markers Pearson Correlation analyses did. As shown in table 3.

There was a positive correlation between serum levels of AST and LDH. A positive relationship was between se- 
Table 2. The skin cancer patient and control group statistics.

\begin{tabular}{|c|c|c|c|c|c|c|c|c|}
\hline & \multicolumn{4}{|c|}{ Patient Group } & \multicolumn{4}{|c|}{ Control Group } \\
\hline & $N$ & Mean & Std. Deviation & Std. Error Mean & $N$ & Mean & Std. Deviation & Std. Error Mean \\
\hline Age & 36 & 59,0556 & 22,46896 & 3,74483 & 40 & 39,5500 & 13,97424 & 2,20952 \\
\hline Glucose $\mathrm{mg} / \mathrm{dl}$ & 36 & 106,5000 & 28,47906 & 4,74651 & 40 & 95,8500 & 11,21023 & 1,77249 \\
\hline Creatinine mg/dl & 36 & 0,9214 & 0,24316 & 0,04053 & 40 & $0,755^{8}$ & 0,16659 & 0,02634 \\
\hline AST U/L & 36 & 22,5278 & 6,70388 & 1,11731 & 40 & 18,1000 & 5,75482 & 0,90992 \\
\hline$A L T U / L$ & 36 & 18,4167 & 6,55689 & 1,09282 & 40 & 32,6500 & 9,84899 & 1,55726 \\
\hline$G G T U / L$ & 27 & 29,5185 & 25,11262 & 4,83293 & 36 & 20,5278 & 10,86406 & 1,81068 \\
\hline Amylase U/L & 25 & 72,2800 & 36,92167 & 7,38433 & 34 & 65,0294 & 24,24807 & 4,15851 \\
\hline$L D H U / L$ & 30 & 258,2000 & 124,52375 & 22,73482 & 35 & 176,1714 & 43,11031 & 7,28697 \\
\hline Biluribin D. mg/dl & 23 & 0,2426 & 0,25234 & 0,05262 & 39 & 0,1328 & 0,05652 & 0,00905 \\
\hline Sodium $\mathrm{mmol} / \mathrm{L}$ & 35 & 139,7143 & 4,42966 & 0,74875 & 35 & 137,6857 & 1,81126 & 0,30616 \\
\hline Potassium mmol/L & 34 & 4,2676 & 0,44635 & 0,07655 & 35 & 4,2086 & 0,46233 & 0,07815 \\
\hline Albumin g/dl & 25 & 4,1920 & 0,46361 & 0,09272 & 32 & 3,9438 & 0,67151 & 0,11871 \\
\hline Bilirubin T. mg/dl & 24 & 0,6992 & 0,33521 & 0,06842 & 40 & 0,5290 & 0,23366 & 0,03695 \\
\hline Phosphormg/dl & 18 & 3,0389 & 0,71549 & 0,16864 & 28 & 3,5643 & 0,53070 & 0,10029 \\
\hline Calcium mg/dl & 30 & 9,0757 & 0,55776 & 0,10183 & 37 & 9,1262 & 0,44774 & 0,07361 \\
\hline Magnesium mg/dl & 19 & 2,1474 & 0,57083 & 0,13096 & 31 & 1,9484 & 0,16707 & 0,03001 \\
\hline Uric Acid mg/dl & 17 & 4,1412 & 1,38251 & 0,33531 & 28 & 4,0143 & 1,10913 & 0,20960 \\
\hline Urea mg/dl & 36 & 34,2444 & 12,36436 & 2,06073 & 40 & 23,5063 & 6,38312 & 1,00926 \\
\hline Chlore $\mathrm{mmol} / \mathrm{L}$ & 22 & 101,6364 & 3,86123 & 0,82322 & 34 & 103,3529 & 1,73873 & 0,29819 \\
\hline
\end{tabular}
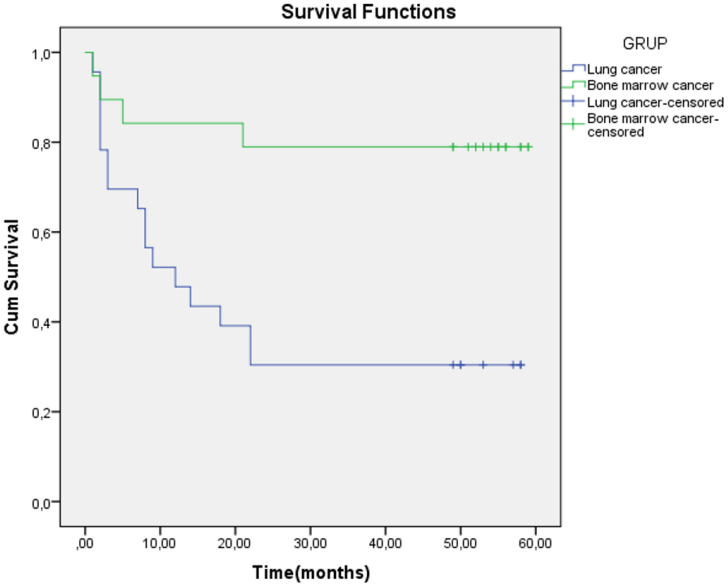

Figure 2. Kaplan-Meier, 5-years survival of lung cancer and bone marrow cancer patients.

rum AST and LDH levels. Their levels increased in a correlatively ( $r=0,490 *$, $p=0,001)$. Fig. 3 .

In this study, there was a positive correlation $(r=0,534 \%$, $\mathrm{p}=0,001)$ between serum levels of ALT and Uric acid. Fig. 4 .

AST and Urea levels were found correlated positively. $(r=0,602 \%, p=0,001)$. Fig. 5 .

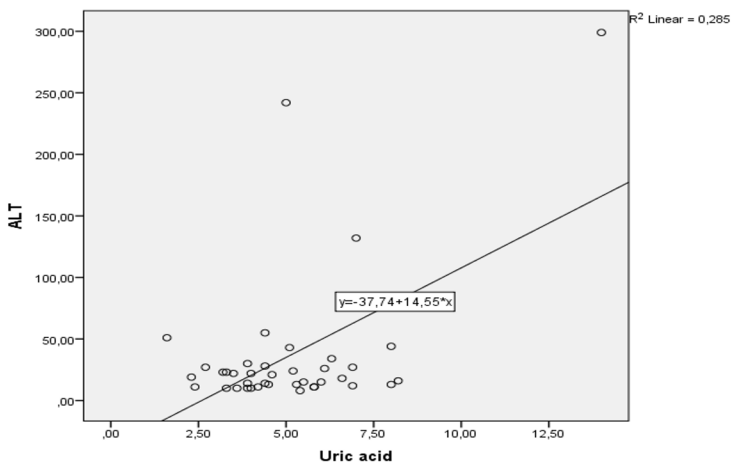

Figure 3. Serum AST and LDH levels correlation.

\section{DISCUSSION}

Risk factors of skin cancers include; having light skin color, excessive sunlight, exposure to X-rays, exposure to chemicals such as coal tar or arsenic, a family history of skin cancer, diseases of genetic repair disorders [13]. When the cases evaluated in terms of incidence, the most common type of cancer was skin cancer (27. 7\%) in Hatay in 2008 [14]. We also detected that the most observed cancer type in Siirt was skin cancer too. The rate of five years in all cancers recorded as $22 \%$ in Siirt. When the 
Table 3. Skin, lung, and bone marrow cancer patients biochemical serum markers Pearson Correlation analysis.

\begin{tabular}{|c|c|c|c|c|c|c|c|c|c|c|}
\hline & & $\begin{array}{l}\text { Creatinin } \\
\mathrm{mg} / \mathrm{dl}\end{array}$ & $\begin{array}{l}A S T \\
U / L\end{array}$ & $\begin{array}{l}A L T \\
U / L\end{array}$ & $\begin{array}{l}L D H \\
U / L\end{array}$ & $\begin{array}{c}\text { Potassium } \\
\mathrm{mmol} / \mathrm{L}\end{array}$ & $\begin{array}{l}\text { Albumin } \\
\text { g/dl }\end{array}$ & $\begin{array}{c}\text { Bilirubin total } \\
\mathrm{mg} / \mathrm{dl}\end{array}$ & $\begin{array}{l}\text { Uric acid } \\
\mathrm{mg} / \mathrm{dl}\end{array}$ & $\begin{array}{l}\text { Urea } \\
m g / d l\end{array}$ \\
\hline \multirow{2}{*}{$\begin{array}{c}\text { Creatinin } \\
m g / d l\end{array}$} & Pearson Correlation & 1 & 169 &, 119 &, 053 & $1333^{* *}$ &,- 184 &, 023 &, $444^{* *}$ &, $563 * *$ \\
\hline & Sig. (2-tailed) & &, 135 & 295 &, 661 &, 003 & 143 & 852 &, 005 &, 000 \\
\hline \multirow{2}{*}{ AST U/L } & Pearson Correlation & 169 & 1 &, $796 * *$ &, $490 * *$ & $299 * *$ &,$- 282 *$ &, 234 & $699 * *$ &, $602 * *$ \\
\hline & Sig. (2-tailed) &, 135 & &, 000 &, 000 &, 008 &, 023 &, 058 &, 000 &, 000 \\
\hline \multirow{2}{*}{$A L T U / L$} & Pearson Correlation & 119 &, $796 * *$ & 1 & $331 * *$ &, 213 & -144 & $257^{*}$ &, $534^{* *}$ &, $498 * *$ \\
\hline & Sig. (2-tailed) &, 295 &, 000 & &, 005 &, 061 &, 254 &, 037 &, 000 &, 000 \\
\hline \multirow{2}{*}{$L D H U / L$} & Pearson Correlation &, 053 &, $490 * *$ & $331 * *$ & 1 &,- 129 &,$- 404 * *$ & $341 * *$ &, $517^{* *}$ &, $430 * *$ \\
\hline & Sig. (2-tailed) &, 661 &, 000 &, 005 & & 287 &, 001 &, 006 &, 001 &, 000 \\
\hline \multirow{2}{*}{$\begin{array}{l}\text { Potassium } \\
\mathrm{mmol} / \mathrm{L}\end{array}$} & Pearson Correlation & $1333^{* *}$ & $299^{* *}$ &, 213 &,- 129 & 1 &, 081 &,- 220 &, $401 *$ & $267 *$ \\
\hline & Sig. (2-tailed) &, 003 &, 008 &, 061 &, 287 & &, 521 &, 076 &, 013 &, 020 \\
\hline \multirow{2}{*}{ Albumin g/dl } & Pearson Correlation &,- 184 &,$- 282 *$ &,- 144 &,$- 404^{* *}$ &, 081 & 1 &,$- 351 * *$ &,$- 477^{* *}$ &,$- 402 * *$ \\
\hline & Sig. (2-tailed) & 143 &, 023 &, 254 &, 001 &, 521 & &, 006 &, 004 &, 001 \\
\hline \multirow{2}{*}{$\begin{array}{c}\text { Bilirubin total } \\
\mathrm{mg} / \mathrm{dl}\end{array}$} & Pearson Correlation &, 023 &, 234 & $257^{*}$ & $341 * *$ &,- 220 & $-351 * *$ & 1 &, 252 & $263^{*}$ \\
\hline & Sig. (2-tailed) & 852 &, 058 &, 037 &, 006 &, 076 &, 006 & &, 165 &, 036 \\
\hline \multirow{2}{*}{$\begin{array}{l}\text { Uric asit } \\
\mathrm{mg} / \mathrm{dl}\end{array}$} & Pearson Correlation &, $444^{* *}$ & $699 * *$ & $1534^{* *}$ & $517^{* *}$ &, $401 *$ &,$- 477 * *$ &, 252 & 1 & $751 * *$ \\
\hline & Sig. (2-tailed) &, 005 &, 000 &, 000 &, 001 &, 013 &, 004 &, 165 & &, 000 \\
\hline \multirow{2}{*}{ Urea $m g / d l$} & Pearson Correlation &, $563 * *$ & $602 * *$ & $498 * *$ &, $430 * *$ & $267 *$ &,$- 402 * *$ & $263^{*}$ &, $751 * *$ & 1 \\
\hline & Sig. (2-tailed) &, 000 &, 000 &, 000 &, 000 &, 020 &, 001 &, 036 &, 000 & \\
\hline
\end{tabular}

**. Correlation is significant at the 0.01 level (2-tailed).

*. Correlation is significant at the 0.05 level (2-tailed).

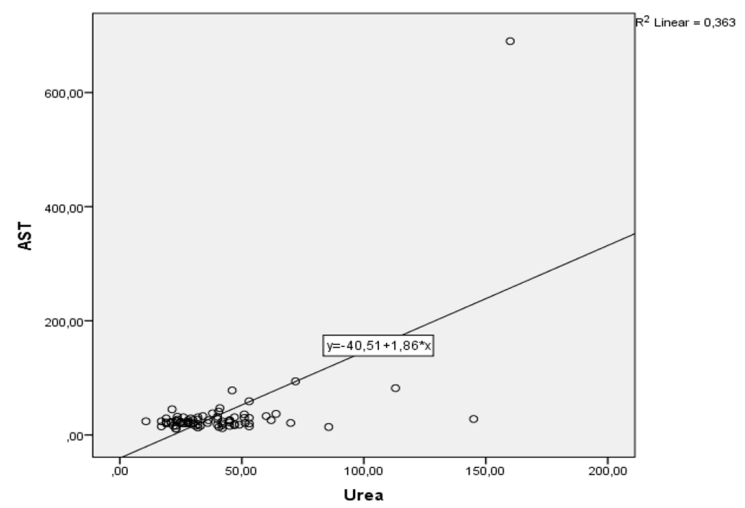

Figure 4. Serum ALT and Uric acid levels correlation.

literature reviewed, skin cancer is more likely to occur in males than in females [14]. In a study in both women and men, the median diagnosis age was 71 years. NMSC is observed most often on the face and body [15]. The prevailing sites opened to strong sunlight, such as hands, the face, neck, and forearms [16]. Our study complies with this study, NMSC has occurred on face site, but the average age of skin cancer in our study was 60 years and low. According to another study, a significant increase in skin cancer in the province of Hatay requires serious attention to environmental factors and genetics. The risk factors for enlargement of skin cancers are going ultraviolet rays, ionizing radiation, human papillomavirus infections, infrared radiation, inorganic arsenic, trauma, immunosupp-

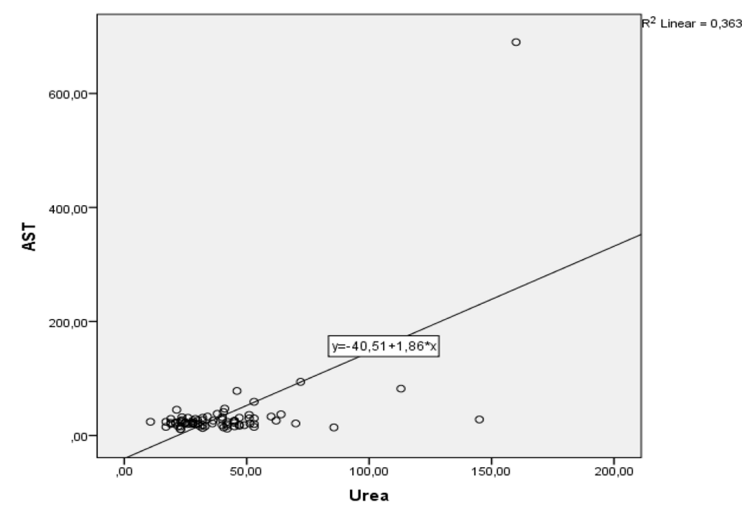

Figure 5. Serum AST and Urea levels correlation.

ression, and genetic diseases. It reported that alcohol and smoking, nutrition, and obesity participated enlargement of various cancers such as skin cancers [14]. For all skin cancers, $60 \%$ of the cases and more were on the regions of skin most opened to the sun, corresponding to the face, scalp, and neck [17]. A rising tendency in inpatient NMSC and melanoma skin cancer of the head and neck region, correlating to an increase in reconstructive procedures applied in the inpatient setting and high expense load [18]. In our study, BCC lesions were commonly observed in the face-cheek region $30 \%$ (29/96), nose $24 \%$ (23/96), and lesions with SCC were most frequently in-ear region 28. 6\% (10/35). Precaution actions such as using a broad-spectrum sunscreen, wearing protective dressing, 
looking for shade, and limiting the time open-air during mid-day hours are very beneficial when started in early life [19]. Increasing the level of education and consciousness of people in less developed countries is crucial for early diagnosis of the patient and timely treatment to decrease the mortality of the illness [20]. From our literature reviews, people should attend to some scientific outcomes to live healthily and especially not get caught skin cancer. We do not recommend anyone go out in the open air between 10 am and $4 \mathrm{pm}$ when the sun's rays are potent. Outdoor activities must perform in the morning or evening. People must use sunscreen even if they have a darker skin tone. Lips, ears, hands, and necks should not forget. Protective clothes must be wear. Tanning beds must be skipped. Medications must be checked. Protective moisturizers and makeup should be used throughout the year. If skin cancers are detected early, they can be treated successfully. People can go to the dermatologist once a year to do a skin cancer scan. It has described that the cancer tissue displayed a high ratio of aerobic glycolysis than other tissues, and it recommends the analysis of biochemical markers in neoplasm. Blood serum; alkaline phosphatase, lactate dehydrogenase, calcium, magnesium, and amylase studied in various cancers as potential diagnostic and prognostic biochemical markers. It was observed that AST and ALT as tumor markers used in the control of malignant of the head and neck and cervix uteri [21]. The serum LDH levels of the skin, lung, and bone marrow patients whose data we examined were statistically significant compared with the control group. A high increase in serum activities of ALT, AST, and GGT was noted in both liver and colon cancer patients relative to the control group and among cancer types. These enzymes can be used as tumor markers in the prognosis, diagnosis, and treatment of cancer, especially in colon and liver cancers [22]. In our study, a significant difference was in these enzyme levels after patients with skin and lung cancer diseases compared with the control group.

In the study based on the province of Siirt, the rate of males was higher than females in terms of lung and bone marrow cancers. Cancer increases with aging. The incidence of these cancers was lower than in western regions. The survival rate of lung cancers was lower than bone marrow cancers. While lung cancer is one of the deadliest cancers with five-year survival rates of at most $15 \%$, the population (80\% of the sample) believe that survival rates exceed $20 \%$ [10]. In 2013, For 5-year survival analysis, the number of patients with lung cancer was 23, and the number of patients with bone marrow cancer was 19.16 (69.6\%) of the lung cancer patients and 4 (21.1\%) of the bone marrow cancer patients died. Two cancer groups were statistically different in terms of 5-years survival. Log Rank X2 $=8.68$, $\mathrm{p}=0.003, \mathrm{p}<0.05$. In this study, there was a statistically significant difference between lung cancer and bone marrow cancer patient's serum glucose, AST, GGT, LDH, urea levels compared with the control group serum parameters level, $\mathrm{p}<0.05$. The Kaplan-Meier test was used for the survival comparison of patients in the two disease groups. There was a significant difference at $\mathrm{p}<0.001$ level between the two groups. Because serum or plasma provides the physiological and pathological state, it offers the most useful studied biological substances for cancer biomarkers [23]. Breast cancer patients with high LDH levels have a 1.42-fold increased risk of death [24]. From our results, LDH and AST can be used as biochemical markers in skin cancers, lung cancers, and bone marrow cancer cases. LDH can be considered a good biomarker for the diagnosis of liver, muscular, and cancer diseases [25]. In this study, a positive correlation found between serum levels of AST and LDH, ALT and Uric acid, and AST and urea. Their levels increased correlatively. In this study, skin cancer patient's serum glucose, AST, ALT, sodium, bilirubin direct, bilirubin total, creatinine, urea level were statistically significantly different compared with the control group's serum parameters levels, $\mathrm{p}<0.05$. Also, a statistically significant difference was between lung cancer and bone marrow cancer's serum glucose, AST, GGT, LDH, Urea levels, and the serum parameters of the control group, $\mathrm{p}<0.05$. For this reason, it is important to study the biochemical parameters found to be statistically significant in all situations that show the symptoms of these diseases and to make their measurements more sensitive.

\section{CONCLUSION}

In conclusion, with our outcomes and the literature, the BCC and SCC observed ratio and the locations of them on the body were significant to comply with the literature and lower even though Siirt were in the Southeast region and takes too much sun in a day. Biochemical serum parameters measured in this article were convenient to investigate and detect skin, lung, and bone marrow, cancer patients. Besides, this study was retrospective, only the recorded in one state hospital data analyzed. Therefore, multicenter studies with large patient populations are also needed.

\section{ACKNOWLEDGEMENT}

This study was supported by Siirt University Scientific Research Projects Coordination (2017-SIÜSYO-66).

\section{CONFLICT OF INTEREST}

Authors approve that to the best of their knowledge, there is not any conflict of interest or common interest with an institution/organization or a person that may affect the review process of the paper. 


\section{AUTHOR CONTRIBUTION}

Hasan Karagecili; Concept, Design, Supervision, Data collection and processing, Analysis, Writing the article, References and fundings

Emrah Yerlikaya; Supervision, Data collection and processing, Analysis, Critical review, References and fundings

Mustafa Oğuzhan Kaya; Supervision, Data collection and processing, Analysis, Literature review, Critical review

\section{References}

1. Nair M, Sandhu SS, Sharma AK. Cancer molecular markers: A guide to cancer detection and management. Semin Cancer Biol 52 (2018) 39-55.

2. Duartea AF, Pinto BS, Freitas A et al. Skin cancer healthcare impact: A nation-wide assessment of an administrative database. Cancer Epidemiol 56 (2018) 154-160.

3. Apalla Z, Lallas A, Sotiriou E et al. Epidemiological trends in skin cancer. Dermatol Pract Concept 7(2) (2017) 1-6.

4. Brunssen A, Waldmann A, Eisemann $\mathrm{N}$ et al. Impact of skin cancer screening and secondary prevention campaigns on skin cancer incidence and mortality: A systematic review. J Am Acad Dermatol 76 (2017) 129-39.

5. O'Sullivan DE, Brenner DR, Demers PA et al. Indoor tanning and skin cancer in Canada: A meta-analysis and attributable burden estimation. Cancer Epidemiol 59 (2019) 1-7.

6. Cho EA, Moloney FJ, Cai H et al. Safety and tolerability of an intratumorally injected DNAzyme, Dz13, in patients with nodular basal-cell carcinoma: a phase 1 first-in-human trial (DISCOVER). Lancet 381 (2013) 1835-43.

7. Chang NB, Feng R, Gao Z et al. Skin cancer incidence is highly associated with ultraviolet-B radiation history. Int J Hyg Envir Heal 213 (2010) 359-368.

8. Didona D, Paolino G, Bottoni U et al. Non Melanoma Skin Cancer Pathogenesis Overview. Biomedicines (2018); 6(1): 6. DOI: 10.3390/ biomedicines6010006.

9. Kansara S, Bell D, Weber R. Surgical management of non melanoma skin cancer of the head and neck. Oral Oncology 100 (2020) 1044853.

10. Ziebarth N.R. Lung cancer risk perception biases. Preventive Medicine 110 (2018) 16-23.

11. Götte M, Kovalszky I. Extracellular matrix functions in lung cancer, Matrix Biol (2017), https://doi.org/10.1016/j.matbio.2018.02.018.

12. Mu C.F, Shen J, Liang J, Zheng H.S, Xiong Y, Wei Y.H, Fanzhu Li. Targeted drug delivery for tumor therapy inside the bone marrow.
Biomaterials 155 (2018) 191-202.

13. Kayabaşoğlu G. An Alternative Flap Approach For Auricle Reconstruction. Sakaryamj 3(1) (2013) 32-35.

14. Arıca S, Nazlıcan E, Özer C et al. The frequency and distribution of cancer cases in Hatay District in 2008. J Clin Exp Invest 2(2) (2011) 192-195.

15. Eisemann N, Waldmann A, Geller AC et al. Non-Melanoma Skin Cancer Incidence and Impact of Skin Cancer Screening on Incidence. J Invest Dermatol 134 (2014) 43-50.

16. Armstrong BK, Cust AE. Sun exposure and skin cancer, and the puzzle of cutaneous melanoma A perspective on Fears et al. Mathematical models of age and ultraviolet effects on the incidence of skin cancer among whites in the United States. American Journal of Epidemiology 1977; 105: 420-427. Cancer Epidemiol 48 (2017) 147-156.

17. Rubió-Casadevall J, Hernandez-Pujol AM, Ferreira-Santos MC et al. Trends in incidence and survival analysis in non-melanoma skin cancer from 1994 to 2012 in Girona, Spain: A population-based study. Cancer Epidemiol 45 (2016) 6-10.

18. Egeler SA, Huang A, Johnson AR et al. Regional incidence of and reconstructive management patterns in melanoma and nonmelanoma skin cancer of the head and neck: A 3-year analysis in the inpatient setting. Journal of Plastic, Reconstructive and Aesthetic Surgery 73 (2020) 507-515.

19. Chougule A, Hussain S, Agarwal DP. Prognostic and diagnostic value of serum pseudocholinesterase serum aspartate transaminase, and serum alanine transaminase in malignancies treated by radiotherapy. J Cancer Res Ther 4(1) (2008) 21-25.

20. Khazaei Z, Ghorat F, Jarrahi AM et al. Global incidence and mortality of skin cancer by histological subtype and its relationship with the human development index (HDI); An Ecology Study in 2018. World Cancer Research Journal 2019; 6: e1265.

21. AL-Janabi, AAHS, Ali ZQ, Noree ZM. Lactate dehydrogenase as an indicator of liver, muscular and cancer diseases. J Coast Life Med 3(7) (2015) 543-546.

22. Ojo O.C, Asaolu M.F, Akinlua I, Oyeyemi A.O, Atiba A.S. Serum Marker Enzymes Activities in Cancer Patients. Journal of Chemistry and Biochemistry December 4(2) (2016) 15-21.

23. Li X. et al. The effect of preoperative serum triglycerides and highdensity lipoprotein-cholesterol levels on the prognosis of breast cancer. The Breast 32 (2017) 1-6.

24. Liu X. et al. Prognostic significance of pretreatment serum levels of albumin, LDH and total bilirubin in patients with nonmetastatic breast cancer Carcinogenesis, 36(2) (2015) 243-248.

25. McRee AL, Mays D, Kornides ML et al. Counseling About Skin Cancer Prevention Among Adolescents: What Do Parents Receive From Health Care Providers? J Adolescent Health 61 (2017) 533-536. 